\title{
Total-internal-reflection Broad-bandwidth Sum Frequency Generation Spectroscopy of Hexadecanethiol Adsorbed on Thin Gold Film Deposited on $\mathrm{CaF}_{2}$
}

\author{
Naoya Nishi, Daisuke Hobara, Masahiro Yamamoto, and Takashi KaKIUCHI ${ }^{\dagger}$ \\ Department of Energy and Hydrocarbon Chemistry, Graduate School of Engineering, Kyoto University, \\ Kyoto 606-8501, Japan
}

\begin{abstract}
Sum frequency vibrational spectra for hexadecanethiol (HDT) adsorbed on thin gold film deposited on the surface of a $\mathrm{CaF}_{2}$ prism have been measured using total-internal reflection broad-bandwidth sum frequency generation (TIR-BBSFG) spectroscopy. The bands attributed to the $\mathrm{CH}_{3}$ symmetric and asymmetric stretching vibrational modes were observed in the sum frequency vibrational spectra. The orientation of the methyl groups was analyzed using the ratio of the intensities of the two modes. The methyl groups of HDT on the thin gold film were much more randomly orientated than those on $\mathrm{Au}(111)$.
\end{abstract}

(Received March 31, 2003; Accepted April 18, 2003)

\section{Introduction}

For the study of surface phenomena at various interfaces, the total internal reflection (TIR) condition has been used in a number of spectroscopic measurements, ${ }^{1}$ such as fluorometry, ${ }^{2-9}$ surface enhanced infrared spectroscopy (SEIRA), ${ }^{10-12}$ second harmonic generation, ${ }^{13-21}$ and sum frequency generation (SFG). ${ }^{22-25}$ There are two advantages when one uses the TIR condition. First, both incident light to the interface and reflected light or generated light from the interface travel in only one of the two phases adjacent to the interface, which circumvents bulk absorption by the other phase. One corollary is the freedom of changing the composition of the adjacent phase that has a lower refractive index. Second, high sensitivity to the interfaces can be achieved owing to the enhancement of the electric field at the interfaces. ${ }^{1}$

The TIR arrangement can be employed at metal interfaces if a very thin metal film is used to avoid the absorption of light. It has been successfully employed to detect adsorbed species on metal in the SEIRA, where the thickness of the metal film is about $10 \mathrm{~nm}$ on a transparent substrate. ${ }^{10-12}$ Recently, Williams et al. showed that the TIR arrangement can also be employed to detect SFG signals from alkanethiols adsorbed on a thin gold film. ${ }^{24}$ However, detailed analysis of the sum frequency vibrational spectra has not been reported. SFG in the TIR arrangement has some potential as a powerful tool to study molecular orientations at metal/liquid interfaces, such as electrochemical interfaces, because it is free from bulk absorption and has interfacial sensitivity in the order of nanometers. Unlike surface plasmon resonance and capacitance measurements that have been used as sensitive means of detecting adsorption and molecular interaction at the interfaces, TIR-SFG will enable us to obtain molecular information on the

† To whom correspondence should be addressed.

E-mail: kakiuchi@scl.kyoto-u.ac.jp solution side of the interfaces. One drawback of the TIR arrangement is the roughness of the metal surfaces. The roughness is prerequisite for high sensitivity and can complicate the interpretation of the experimental data because of the orientational distribution of molecules in comparison with those adsorbed on a single crystal surface. It is therefore of crucial importance to assess the degree of the variation in orientation of molecules.

By using broad-bandwidth sum frequency generation $(\mathrm{BBSFG})^{26-28}$ we recently analyzed the orientation of alkanethiols adsorbed on $\mathrm{Au}(111) .{ }^{29}$ It is worthwhile to examine the applicability of BBSFG in the TIR condition at metal interfaces to obtain SFG spectra with higher resolution. In the present paper, we report the results of TIR-BBSFG measurements for hexadecanethiol (HDT) adsorbed on a thin gold film on $\mathrm{CaF}_{2}$. We will show that BBSFG can be successfully employed in the orientation analysis of the HDT molecules and that the methyl groups of HDT are much more randomly orientated than those on $\mathrm{Au}(111)$ on mica.

\section{Experimental}

\section{Substrate}

A hemicylindrical $\mathrm{CaF}_{2}$ prism (Pier Optics, Japan) was cleaned in $\mathrm{KOH}$-saturated ethanol and then washed with Milli$Q$ water before use. Gold was vapor-deposited on the basal plane of the hemicylindrical prism under the pressure of $1.3 \times$ $10^{-3} \mathrm{~Pa}$ at room temperature. The evaporation rate and the thickness of gold were monitored by a quartz crystal microbalance, and were $0.03 \mathrm{~nm} \mathrm{~s}^{-1}$ and $10 \mathrm{~nm}$, respectively. The evaporation conditions affected the morphology of thin metal films and were critical for enhancing the electric field at adsorbate/metal interfaces, as suggested by Nishikawa et al. using $\mathrm{BaF}_{2}$ and $\mathrm{Ge}$ prisms. ${ }^{11}$ In the deposition, we followed their conditions so that separated metal islands can be formed on the substrate surface. For the external reflection (ER) 


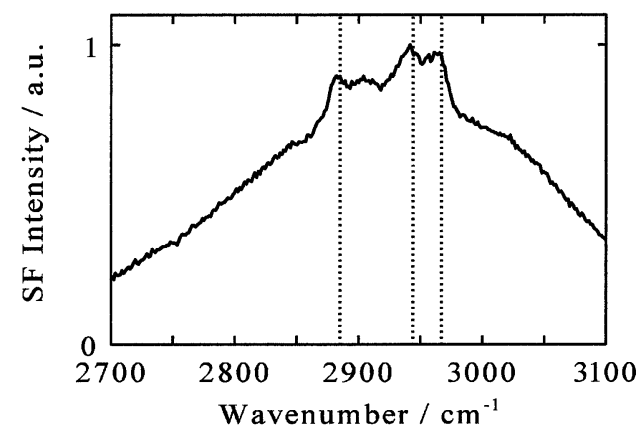

Fig. 1 Sum frequency vibrational spectrum for HDT adsorbed on the $10 \mathrm{~nm}$ gold film on $\mathrm{CaF}_{2}$ in the TIR condition. The three dotted lines are 2885, 2944, and $2967 \mathrm{~cm}^{-1}$, respectively.

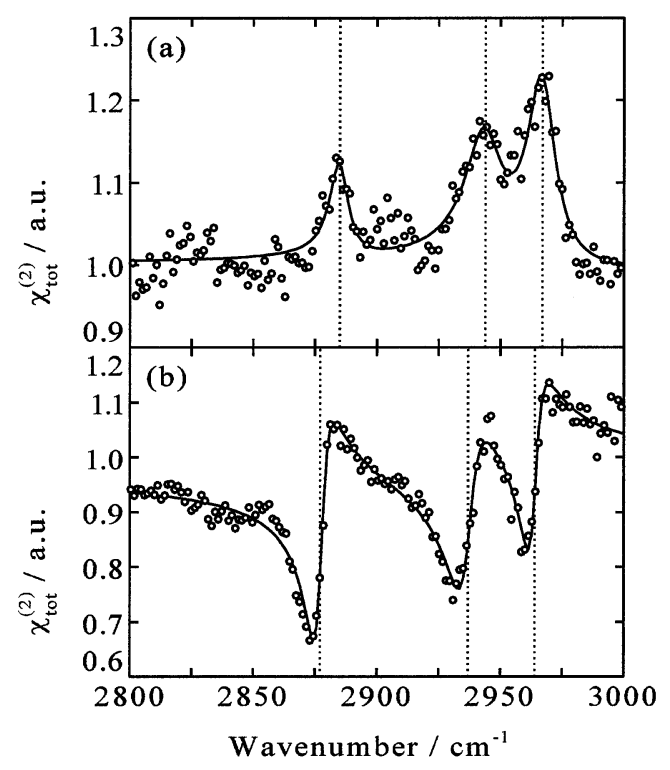

Fig. 2 Measured $\chi_{\text {tot }}^{(2)}$ for HDT adsorbed on the $10 \mathrm{~nm}$ gold film on $\mathrm{CaF}_{2}$ in the TIR condition and (b) for HDT adsorbed on $\mathrm{Au}(111)$ in the ER condition. ${ }^{29}$ The solid lines show least-square fits to the data. The three dotted lines in (a) are 2885, 2944, and $2967 \mathrm{~cm}^{-1}$, and those in (b) are 2877,2937 , and $2964 \mathrm{~cm}^{-1}$, respectively.

condition, $200 \mathrm{~nm}$ thickness gold deposited on mica was prepared as described elsewhere. ${ }^{30}$ Both substrates were immersed in $1 \times 10^{-3} \mathrm{~mol} \mathrm{dm}^{-3}$ ethanol solution of HDT (TCI, Japan) and held in the solution at least $12 \mathrm{~h}$ before measurements.

\section{BBSFG system}

Both broad-bandwidth IR pulses (duration-150 fs, bandwidth$200 \mathrm{~cm}^{-1}$, energy-1.5 $\mu \mathrm{J} /$ pulse) and narrow-bandwidth visible pulses (duration-10 ps, bandwidth-12 $\mathrm{cm}^{-1}$, energy- $2.5 \mu \mathrm{J} / \mathrm{pulse}$ ) were made collinear and were introduced to the gold surfaces. In the TIR condition, pulses were introduced to the thiolcovered gold surface on the basal plane of the $\mathrm{CaF}_{2}$ prism from the $\mathrm{CaF}_{2}$ side. In the $\mathrm{ER}$ condition, the $\mathrm{Au}(111)$ surface on mica was irradiated from the air side. The incident angle was $60^{\circ}$ in both cases. Both IR and visible pulses were $p$-polarized and the $p$-polarized component of sum-frequency (SF) light was analyzed. The SF light with broad-bandwidth was dispersed by a monochromator and each wavelength component was simultaneously detected by a CCD. Other details have been described elsewhere..$^{29}$

\section{Results and Discussion}

TIR-BBSFG

The dependence of the SF intensity, $I_{\mathrm{sf}}$, on the angular frequency of IR pulses, $\omega_{\mathrm{ir}}$, may be described by: ${ }^{31}$

$$
I_{\mathrm{sf}}\left(\omega_{\mathrm{ir}}\right)=\left|\chi_{\mathrm{tot}}^{(2)}\left(\omega_{\mathrm{ir}}\right)\right|^{2} I_{\mathrm{ir}}\left(\omega_{\mathrm{ir}}\right) I_{\mathrm{vis}}
$$

where $\chi_{\text {tot }}^{(2)}$ is the second-order susceptibility, and $I_{\text {ir }}$ and $I_{\mathrm{vis}}$ are the intensities of IR and visible pulses, respectively. The second-order susceptibility can be divided into two components, i.e., the nonlinear susceptibility of gold, $\chi_{\mathrm{NR}}^{(2)}$, and the resonant susceptibility, $\chi_{R, v}^{(2)}$, of a vibrational mode, $v::^{22,33}$

$$
\begin{aligned}
\chi_{\mathrm{tot}}^{(2)} & =\chi_{\mathrm{NR}}^{(2)}+\sum_{v} \chi_{\mathrm{R}, v}^{(2)} e^{i \phi v} \\
& =\chi_{\mathrm{NR}}^{(2)}\left(1+\sum_{v} \frac{A_{v} e^{i \phi v}}{\omega_{\mathrm{ir}}-\omega_{v}+i \Gamma_{v}}\right)
\end{aligned}
$$

where $A_{v}, \omega_{v}$, and $\Gamma_{v}$ are the amplitude, the angular frequency, and the bandwidth of the Lorentzian function for $v$, respectively. ${ }^{31}$ It is noted that $\chi_{\mathrm{NR}}^{(2)}$ and $\chi_{\mathrm{R}, v}^{(2)}$ contain the Fresnel coeffcients, ${ }^{24,28}$ and that $A_{v}$ is normalized by $\chi_{\mathrm{NR}}^{(2)}$. For the orientation analysis of the methyl groups, the vibrational intensity of $v, V_{v}$, may be defined as follows: ${ }^{34}$

$$
\begin{aligned}
V_{v} & \equiv\left|\frac{\chi_{\mathrm{R}, v}^{(2)}\left(\omega_{\mathrm{ir}}=\omega_{v}\right)}{\chi_{\mathrm{NR}}^{(2)}}\right| \\
& =A_{v} / \Gamma_{v}
\end{aligned}
$$

Figure 1 shows an SF vibrational spectrum for the HDTadsorbed $10 \mathrm{~nm}$ thickness thin gold film deposited on the $\mathrm{CaF}_{2}$ prism in the TIR condition. A broad envelope in the spectrum can be assigned to the intraband transition of gold that causes $\chi_{\mathrm{NR}}^{(2)}$. The envelope corresponds to that of broad-bandwidth IR pulses. In the envelope three peaks were detected at 2885, 2944 and $2967 \mathrm{~cm}^{-1}$; these can be assigned to the $\mathrm{CH}_{3}$ symmetric stretching $\left(\mathrm{r}^{+}\right)$, the Fermi resonance between $\mathrm{r}^{+}$and the overtone of the $\mathrm{CH}_{3}$ bending $\left(\mathrm{r}_{\mathrm{FR}}^{+}\right.$), and the $\mathrm{CH}_{3}$ asymmetric stretching $(r)$, respectively. We observed three dips in the ER condition rather than peaks. ${ }^{29}$ This difference in $\phi$ results from both the change in the Fresnel coeffcients ${ }^{35,36}$ and the contribution of the gold/ $\mathrm{CaF}_{2}$ interface to $\chi_{\mathrm{NR} .}^{(2)}{ }^{24}$ We normalized this spectrum by that for a GaAs surface; the susceptibility of the GaAs surface is independent of the wavelength in the region studied and we hence can measure the IR envelope ${ }^{37}$ The plot in Fig. 2a shows the normalized spectrum, in which the three peaks become clearer. In Fig. 2a, a small band can be seen at $\sim 2910 \mathrm{~cm}^{-1}$, corresponding to the methylene asymmetric stretching, which is SFG-active when the methylene groups are in gauche conformation. ${ }^{24,40}$ This indicates that a detectable amount of gauche conformations is present, unlike alkanethiols adsorbed on $\mathrm{Au}(111)$-prevalent gold surface.

Comparison of parameters from TIR-BBSFG with those from ER-BBSFG

The vibrational intensities of the $\mathrm{CH}_{3}$ stretching vibrational modes reflect the degree of the orientation of the methyl groups with respect to the surface normal. ${ }^{29,38,39}$ To obtain the vibrational intensities and other parameters, Eq. (2) was fitted to the plot in Fig. 2a, taking account of a linear baseline. The 
Table 1 Fitted parameters for the bands in the SF vibrational spectra for HDT adsorbed on gold using $\mathrm{TIR}^{\mathrm{a}}$ and $\mathrm{ER}^{\mathrm{b}}$ conditions

\begin{tabular}{ccccccc}
\hline Condition & Mode & $A / \mathrm{cm}^{-1}$ & $\phi / \pi \mathrm{rad}$. & $v_{0} / \mathrm{cm}^{-1}$ & $\Gamma / \mathrm{cm}^{-1}$ & $V(=A / \Gamma)$ \\
\hline TIR $^{\mathrm{a}}$ & $\mathrm{r}^{\mathrm{+c}}$ & 0.25 & $0.55^{\mathrm{f}}$ & 2885 & 4.6 & 0.055 \\
& $\mathrm{r}_{\mathrm{FR}}^{+\mathrm{d}}$ & 0.62 & $0.55^{\mathrm{f}}$ & 2944 & 9.0 & 0.069 \\
& $\mathrm{r}^{\mathrm{e}}$ & 0.73 & $0.55^{\mathrm{f}}$ & 2968 & 6.9 & 0.106 \\
$\mathrm{ER}^{\mathrm{b}}$ & $\mathrm{r}^{\mathrm{+c}}$ & 1.15 & 1.85 & $2877^{\mathrm{h}}$ & $4^{\mathrm{h}}$ & 0.288 \\
& $\mathrm{r}_{\mathrm{FR}}^{+\mathrm{d}}$ & 1.24 & $1.84^{\mathrm{g}}$ & $2937^{\mathrm{h}}$ & $6^{\mathrm{h}}$ & 0.207 \\
& $\mathrm{r}^{-\mathrm{e}}$ & 0.89 & $1.84^{\mathrm{g}}$ & $2964^{\mathrm{h}}$ & $4^{\mathrm{h}}$ & 0.221 \\
\hline
\end{tabular}

a. HDT adsorbed on the $10 \mathrm{~nm}$ thin gold film on $\mathrm{CaF}_{2}$

b. HDT adsorbed on $\mathrm{Au}(111)$ on mica. ${ }^{29}$

c,d. The $\mathrm{CH}_{3}$ symmetric stretching (split bands due to the Fermi resonance).

e. The $\mathrm{CH}_{3}$ asymmetric stretching.

f,g. Forced to be equal.

h. Fixed parameters.

Table 2 Orientation of the methyl groups of HDT adsorbed on gold using $\mathrm{TIR}^{\mathrm{a}}$ and $\mathrm{ER}^{\mathrm{b}}$ conditions

\begin{tabular}{cccccc}
\hline Condition & $V_{\mathrm{r}+\mathrm{u}}$ & $V_{\mathrm{r}-}$ & $V_{\mathrm{r}-} / V_{\mathrm{r}+\mathrm{u}}$ & $\theta^{\mathrm{c}} / \mathrm{deg}$ & $\Delta \theta^{\mathrm{d}} / \mathrm{deg}$ \\
\hline TIR $^{\mathrm{a}}$ & 0.088 & 0.106 & 1.20 & 36 & 0 \\
& & & & 25 & 50 \\
ER $^{\mathrm{b}}$ & 0.354 & 0.221 & 0.62 & 25 & 0 \\
\hline
\end{tabular}

a. HDT adsorbed on the $10 \mathrm{~nm}$ thin gold film on $\mathrm{CaF}_{2}$.

b. HDT adsorbed on $\mathrm{Au}(111)$ on mica. ${ }^{29}$

c. Angle between the main axis of the methyl group and the surface normal.

d. FWHM of $\theta$.

measured susceptibility from HDT adsorbed on $\mathrm{Au}(111)$ in the ER condition is shown in Fig. 2 b for comparison. ${ }^{29}$ Table 1 summarizes the parameters obtained for the two cases. The position of the bands in the TIR condition is $4-8 \mathrm{~cm}^{-1}$ more positive than that in the ER condition. The position of $\mathrm{r}^{+}$in IR reflection-absorption spectra of alkanethiols on gold is known to positively shift with an increase in temperature, reflecting the increasingly disordered local environment around the methyl groups. ${ }^{41,42}$ Thus the methyl groups of the HDT molecules on the thin gold film seem to be more disordered compared with those on $\mathrm{Au}(111)$, owing to the gauche conformations of the alkyl chain terminal of HDT. The greater $\Gamma$ in the TIR condition supports this interpretation. The values of $V$ in the TIR condition are less than a half of those in the ER condition. The decrease can be explained as follows. First, the $\mathrm{CaF}_{2} /$ gold interface as well as the thiol-covered gold surface contributes to $\chi_{\mathrm{NR}}^{(2)}$. Second, the SF light generated at the gold/thiol surface is probably attenuated by absorption by gold on the way to the $\mathrm{CaF}_{2}$ phase. Other factors such as the change in the $\chi_{\mathrm{NR}}^{(2)}$ and $\chi_{\mathrm{R}}^{(2)}$ magnitudes compared with HDT adsorbed on Au(111) may contribute to the decrease.

Orientation analysis of the HDT molecules on the thin gold film

To analyze the orientation of HDT adsorbed on the thin gold film, the ratio of the vibrational intensities was used as our previous report in the ER condition. ${ }^{29}$ Though the values of $V$ are underestimated in the TIR condition, the ratio is expected to remain unchanged; $\chi_{\mathrm{NR}}^{(2)}$ is canceled out in the ratio. Since $\mathrm{r}^{+}$is split owing to the Fermi resonance, we evaluated the unperturbed vibrational intensity, $V_{\mathrm{r}+, \mathrm{u}}$, using the following equation: ${ }^{43,44}$

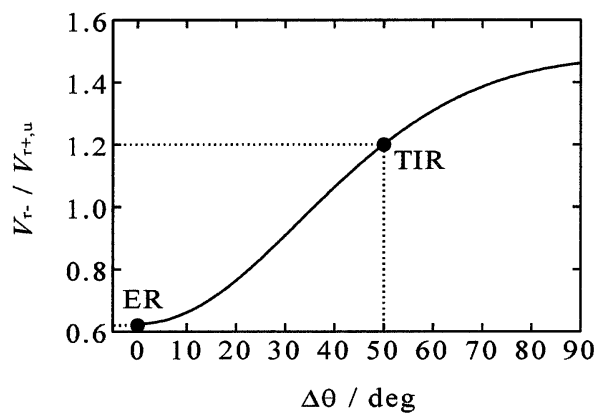

Fig. 3 Dependence of the ratio of the vibrational intensities, $V_{\mathrm{r}\lrcorner} / V_{\mathrm{r}+, \mathrm{u}}$, on the FWHM of the $\theta$ distribution, $\Delta \theta$, when $\theta_{0}=25^{\circ}$.

$$
V_{\mathrm{r}+, \mathrm{u}}=\left(V_{\mathrm{r}+, 1^{2}}+V_{\mathrm{r}+2^{2}}\right)^{1 / 2}
$$

where $V_{\mathrm{r}+1,1}$ and $V_{\mathrm{r}+, 2}$ are the vibrational intensities of 2885 and $2944 \mathrm{~cm}^{-1}$ bands. The ratio of the vibrational intensities of $V_{\mathrm{r}-}$ and $V_{\mathrm{r}+\mathrm{u}}$ can be approximated by: ${ }^{29}$

$$
V_{\mathrm{r}-} / V_{\mathrm{r}+, \mathrm{u}}=D\left(\frac{\langle\cos \theta-\cos 3 \theta>}{\langle\cos \theta>}\right)
$$

where $\theta$ is the angle between the main axis of the methyl group and the surface normal, $D$ is a constant including the hyperpolarizabilities of a methyl group, and the brackets represent the ensemble average over the orientational distribution. Since the nonlinear susceptibility of gold, $\chi_{\mathrm{NR}}^{(2)}$, is canceled out in the ratio, we can analyze the orientation of methyl groups regardless of the TIR or ER conditions.

Using $D=0.88$ from our previous report ${ }^{29,45}$ and assuming no distribution of the orientation, we evaluated each value of $\theta$ for TIR and ER conditions (Table 2). In the ER condition, $\theta=25^{\circ}$, which corresponds to the expected angle $27^{\circ}$ when the all-trans alkyl chain of HDT is $30^{\circ}$ tilted and $50^{\circ}$ rotated. ${ }^{29}$ On the other hand, the value in the TIR condition is $36^{\circ}$, significantly greater than $25^{\circ}$ in the ER condition. This discrepancy may be caused by the assumption of no orientational distribution of the methyl groups in the TIR condition. The surface of the thin gold film may be rough and consist of many separated islands. ${ }^{11}$ The positive shift of the wavenumbers and the greater values of $\Gamma$ in Table 1 support the presence of the orientational distribution of the methyl groups. We estimated the degree of the orientational distribution in the TIR condition by assuming a simple Gaussian form:

$$
f(\theta)=\sin \theta \exp \left[-4 \ln 2 \times\left\{\left(\theta-\theta_{0}\right) / \Delta \theta\right\}^{2}\right]
$$

where $f(\theta)$ is the distribution function of $\theta, \theta_{0}$ is the center of the distribution, and $\Delta \theta$ is FWHM. Figure 3 shows the dependence of $V_{\mathrm{r}-} / V_{\mathrm{r}+\mathrm{u}}$ on $\Delta \theta$ when $\theta_{0}=25^{\circ}$. The $V_{\mathrm{r}-} / V_{\mathrm{r}+\mathrm{u}}$ value of 1.20 in the TIR condition corresponds to $\Delta \theta=50^{\circ}$. This large distribution presumably reflects the morphology of the surface of the thin gold film, in addition to the gauche conformations described above. We further evaluated the value of $V_{\mathrm{r}-} / V_{\mathrm{r}+, \mathrm{u}}$ when a random distribution of $\theta$ (i.e., $f(\theta)=\sin \theta$ ) is assumed in the range of $0^{\circ}<\theta<90^{\circ}$, and found the value, 1.75, which is considerably greater than 1.20 in the TIR condition. We conclude therefore that the methyl groups of the HDT molecules on the thin gold film are much more randomly orientated than those on $\mathrm{Au}(111)$, yet the former still retain a preferential orientation around $\theta=25^{\circ}$. 


\section{Conclusion}

TIR-BBSFG was applied to detect hexadecanethiol molecules adsorbed on a thin gold film deposited on a calcium fluoride. The hexadecanethiol molecules on the thin gold film are much more randomly oriented than those on $\mathrm{Au}(111)$, indicating that the distribution of molecular orientations on thin metals should be taken into account for the orientation analysis. Nevertheless, TIR-BBSFG is promising for in situ measurements of molecules adsorbed on metal surfaces in contact with a solution, as is the case of electrochemical systems.

\section{Acknowledgements}

This work was partially supported by a Grant-in-Aid for Scientific Research (No. 14205120) from the Ministry of Education, Science, Sports, and Culture, Japan, and by CREST of JST (Japan Science and Technology). The SFG system employed in the present study is part of the IRPAF (Interfacial Reaction Properties Analysis Facilities) in Department of Energy and Hydrocarbon Chemistry, Graduate School of Engineering, Kyoto University. This work was conducted under the program, the Center of Excellence for United Approach to New Materials Science, Kyoto University.

\section{References}

1. N. J. Harrick, "Internal Reflection Spectroscopy", 1967, Wiley, New York.

2. D. Axelrod, T. P. Burghardt, and N. L. Thompson, Ann. Rev. Biophys. Bioeng., 1984, 13, 247.

3. M. J. Wirth and J. D. Burbage, J. Phys. Chem., 1992, 96, 9022.

4. T. Kakiuchi, Y. Takasu, and M. Senda, Anal. Chem., 1992, 64, 3096.

5. Y. Saitoh and H. Watarai, Bull. Chem. Soc. Jpn., 1997, 70, 351.

6. S. Ishizaka, K. Nakatani, S. Habuchi, and N. Kitamura, Anal. Chem., 1999, 71, 419.

7. M. A. Jones and P. W. Bohn, Anal. Chem., 2000, 72, 3776.

8. H. Nagatani, R. A. Iglesias, D. J. Fermíin, P.-F. Brevet, and H. H. Girault, J. Phys. Chem. B, 2000, 104, 6869.

9. N. Nishi, K. Izawa, M. Yamamoto, and T. Kakiuchi, J. Phys. Chem. B, 2001, 105, 8162.

10. A. Hartstein, J. R. Kirtley, and J. C. Tsang, Phys. Rev. Lett., 1980, 45, 201.

11. Y. Nishikawa, T. Nagasawa, K. Fujiwara, and M. Osawa, Vib. Spectrosc., 1993, 6, 43.

12. M. Osawa, Bull. Chem. Soc. Jpn., 1997, 70, 2861.

13. N. Bloembergen, H. J. Simon, and C. H. Lee, Phys. Rev., 1969, 181, 1261.

14. G. Berkovic, Y. R. Shen, G. Marowsky, and R. Steinhoff, J. Opt. Soc. Am. B, 1989, 6, 205.

15. B. U. Felderhof, A. Bratz, G. Marowsky, O. Roders, and F. Sieverdes, J. Opt. Soc. Am. B, 1993, 10, 1824.

16. J. C. Conboy, J. L. Daschbach, and G. L. Richmond, J. Phys. Chem., 1994, 98, 9688.
17. A. A. Tamburello Luca, P. Hébert, P.-F. Brevet, and H. H. Girault, J. Chem. Soc., Faraday Trans., 1995, 91, 1763.

18. H. J. Paul and R. M. Corn, J. Phys. Chem. B, 1997, 101, 4494.

19. A. Piron, P. F. Brevet, and H. H. Girault, J. Electroanal. Chem., 2000, 483, 29.

20. T. Uchida, A. Yamaguchi, T. Ina, and N. Teramae, J. Phys. Chem. B, 2000, 104, 12091.

21. X. Shang, A. V. Benderskii, and K. B. Eisenthal, J. Phys. Chem. B, 2001, 105, 11578.

22. S. R. Hatch, R. S. Polizzotti, S. Dougal, and P. Rabinowitz, Chem. Phys. Lett., 1992, 196, 97.

23. M. C. Messmer, J. C. Conboy, and G. L. Richmond, J. Am. Chem. Soc., 1995, 117, 8039.

24. C. T. Williams, Y. Yang, and C. D. Bain, Langmuir, 2000, 16, 2343.

25. G. L. Richmond, Ann. Rev. Phys. Chem., 2001, 52, 357.

26. E. W. M. van der Ham, Q. H. F. Vrehen, and E. R. Eliel, Opt. Lett., 1996, 21, 1448.

27. L. J. Richter, T. P. Petralli-Mallow, and J. C. Stephenson, Opt. Lett., 1998, 23, 1594.

28. R. Braun, B. D. Casson, C. D. Bain, E. W. M. van der Ham, Q. H. F. Vrehen, E. R. Eliel, A. M. Briggs, and P. B. Davies, J. Chem. Phys., 1999, 110, 4634.

29. N. Nishi, D. Hobara, M. Yamamoto, and T. Kakiuchi, J. Chem. Phys., 2003, 118, 1904.

30. T. Kakiuchi, H. Usui, D. Hobara, and M. Yamamoto, Langmuir, 2002, 18, 5231.

31. Y. R. Shen, "The Principles of Nonlinear Optics", 1984, Wiley, New York, 83.

32. J. Miragliotta, R. S. Polizzotti, P. Rabinowitz, S. D. Cameron, and R. B. Hall, Chem. Phys., 1990, 143, 123.

33. C. D. Bain, P. B. Davies, T. H. Ong, R. N. Ward, and M. A. Brown, Langmuir, 1991, 7, 1563.

34. M. S. Yeganeh, S. M. Dougal, R. S. Polizzotti, and P. Rabinowitz, Phys. Rev. Lett., 1995, 74, 1811.

35. T. F. Heinz, in "Nonlinear Surface Electromagnetic Phenomena", ed. H.-E. Ponath and G. I. Stegeman, 1991, Elsevier Science, Amsterdam, 353.

36. C. Hirose, N. Akamatsu, and K. Domen, Appl. Spectrosc., 1992, 46, 1051.

37. T. Ishibashi and H. Onishi, Chem. Phys. Lett., 2001, 346, 413.

38. G. R. Bell, C. D. Bain, and R. N. Ward, J. Chem. Soc., Faraday Trans., 1996, 92, 515.

39. M. S. Johal, E. W. Usadi, and P. B. Davies, J. Chem. Soc., Faraday Trans., 1996, 92, 573.

40. J. Thome, M. Himmelhaus, M. Zharnikov, and M. Grunze, Langmuir, 1998, 14, 7435.

41. R. G. Nuzzo, E. M. Korenic, and L. H. Dubois, J. Chem. Phys., 1990, 93, 767.

42. F. Bensebaa, T. H. Ellis, A. Badia, and R. B. Lennox, Langmuir, 1998, 14, 2361.

43. M. Epple, A. M. Bittner, K. Kuhnke, K. Kern, W.-Q Zheng, and A. Tadjeddine, Langmuir, 2002, 18, 773.

44. N. Nishi, D. Hobara, M. Yamamoto, and T. Kakiuchi, Langmuir, submitted.

45. In Ref. 29 , the $D$ value of 3.5 should be read as 0.88 (= $3.5 / 4)$. 